\title{
3D Segmentation of the Left Ventricle combining Long- and Shortaxis Views
}

\author{
Jatin Relan ${ }^{1}$, Dennis Säring ${ }^{1}$, Michael Groth ${ }^{2}$, Kai Müllerleile ${ }^{3}$, Heinz Handels ${ }^{1}$ \\ ${ }^{1}$ Department of Medical Informatics \\ ${ }^{2}$ Department of Diagnostic and Interventional Radiology \\ ${ }^{3}$ Department of Cardiology/Angiology \\ University Medical Center Hamburg-Eppendorf, 20246 Hamburg, Germany \\ jatinralhan@hotmail.com
}

\begin{abstract}
Segmentation of the left ventricle (LV) is required to quantify LV remodeling after myocardial infarction. Therefore spatiotemporal Cine MR sequences including longaxis and shortaxis images are acquired. In this paper a new segmentation method for fast and robust segmentation of the left ventricle is presented. The new approach considers the position of the mitral valve and the apex as well as the longaxis contours to generate a 3D LV surface model. The segmentation result can be checked and adjusted in the shortaxis images. Finally quantitative parameters were extracted. For evaluation the LV was segmented in eight datasets of the same subject by two medical experts using a contour drawing tool and the new segmentation tool. The results of both methods were compared concerning interaction time and intra- and interobserver variance. The presented segmentation method proved to be fast. The intra- and interobserver variance is decreased for all extracted parameters.
\end{abstract}

\section{Introduction}

Cardiovascular disease is reported as the number one cause of death globally. Approximately 280,000 Germans suffer from a myocardial infarction each year. The infarcted area of the myocardium loses its ability to contract and the remaining healthy muscle needs to compensate for that weakened area. This yields to left ventricle (LV) remodeling which is characterized by e.g. decreased LV ejection fraction. The quantification of LV remodeling based on volume and mass paramters is an indicator for diagnosis and treatment planing. Säring [1] (2006) presented a software system HeAT ( analysis of $4 \mathrm{D}$ MR image sequences. The analysis was based on a manual LV segmentation where the user has to define endocardial and epicardial structures in all shortaxis images. This segmentation process is time-consuming and observerdependent.

In this paper a new semi-automatic segmentation method for the detection of myocardial structure combining longaxis (LA) and shortaxis (SA) images is presented. Recently several approaches for the LV segmentation were published. These approaches can be divided in intensity, shape and model based methods 
$[2,3]$. Generally these methods operate on shortaxis images (Fig. 1(c)) only. Due to the slice thickness of $10 \mathrm{~mm}$ and the orientation of SA images in clinical Cine MR datasets the definition of the ending (mitral valve) slice is ambiguous. So the number of slices for calculating the volume or mass parameters are userdependent. Therefore longaxis views (Fig. $1(\mathrm{a}+\mathrm{b})$ ) have to be included to identify the position of the mitral valve plane in 3D space. Goshtaby et al. [4] show the fusing of LA and SA images into an isotropic volume image using voxel interpolation without posterior LV segmentation. In van Geuns et al. [5] SA contours are generated based on LA contours using spline interpolation. Here the global parameters are computed using the Simpson rule on the number of SA contours without including information concerning mitral valve position.

\section{Materials and Methods}

The goal of this work is to develop a fast segmentation method with reduced interobserver variability. Therefore LA information about position and orientation of the mitral valve plane $(M V P)$ and the apex as well as the myocardial contours (endocardium, epicardium) are used to generate a 3D surface model of the left ventricle. Due to the fact that only $4 \mathrm{LA}$ contours have to be outlined the time for the segmentation process will be decreased. In SA images the shape of the model can be visually inspected and if needed manually adjusted.

\subsection{Material}

Unfortunately no gold standard for LV segmentation exists and software phantoms are commonly used to evaluate new methods [6]. In this paper four datasets $\left(D_{1,2,3,4}\right)$ from the same subject but in different subject position are acquired. The slice thickness $s$ is changed in comparison to the clinical standard $\left(D_{1,2}: s=\right.$ $6 \mathrm{~mm}$, no gap; $D_{3}: s=8 \mathrm{~mm}$, gap $=2 \mathrm{~mm} ; D_{4}: s=10 \mathrm{~mm}$, gap $=2 \mathrm{~mm}$; standard: $s=8 \mathrm{~mm}$, gap $=2 \mathrm{~mm})$. Now two of these datasets $D_{k}$ with $(k=1,2)$ were divided into subdatasets with different number of slices and different startslices and different $s$.

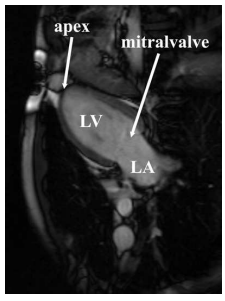

(a)

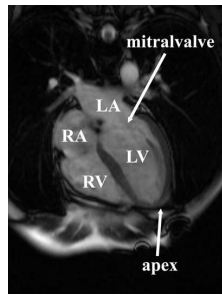

(b)

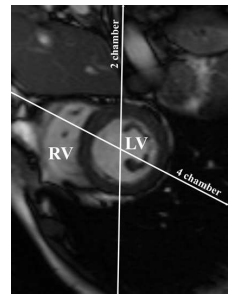

(c)

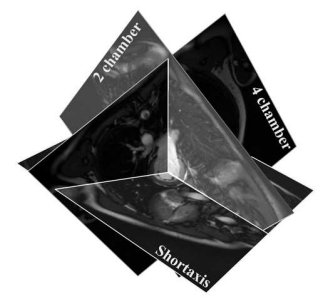

(d)

Fig. 1. 4D Cine MR sequence in vertical longaxis (a) and horizontal longaxis (b) and a midventricular shortaxis (c) 2D views and transferred in 3D coordinate system (d). (LV: left ventricle; LA: left atrium; RV: right ventricle; RA: right atrium) 
- $D_{k}^{1}$ is build by appending all odd numbered slices of $D_{k}$

- $D_{k}^{2}$ is build by appending all even numbered slices of $D_{k}$

Optimally the calculated volume and mass parameters for all datasets are equal. $D_{1,2,3,4}$ are acquired in vertical longaxis (2 chamber view) and horizontal longaxis (4 chamber view) and shortaxis ( $\mathrm{SA})$ views.

\subsection{Methods}

In this paper LA and SA images are transferred from 2D in a 3D coordinate system based on the DICOM header information solving a linear equation [4] (Fig. 1(c)). Mitral valve and apex as well as endocardial and epicardial contours are outlined in vertical and horizontal LA $2 \mathrm{D}$ view (Fig. 2(a)). The mitral valve plane $(M V P)$ is build in 3D using position information of both LA views. Then intersection planes $\left(I S P_{i}\right.$ with $\left.i=1, \ldots, n\right)$ with a distance of $1 \mathrm{~mm}$ are generated automatically parallel to the $M V P$ covering the whole left ventricle from apex to mitral valve (Fig. 2(b)). For each plane $I S P_{i}$ the four endocardial $e n d o_{i}^{j}$ and four epicardial $e p i_{i}^{j}(j=1, \ldots, 4)$ intersection points are calculated (Fig. 2(c)). Defining these intersection points as seedpoints enable the generation of smooth in-plane contours $C_{i}^{e n d o}$ and $C_{i}^{e p i}$ using Bezier interpolation. 3D surface models for endocardium and epicardium are constructed based on LA contours and all in-plane contours $C_{i}^{e n d o}$ and $C_{i}^{e p i}$ for $i=1, \ldots, n$. Additionally contours in the original shortaxis views are extracted by cutting the surface models in 3D space with the image plane. These contours can be reviewed and adjusted. Mass and volume parameters are calculated using the adjusted 3D surface models.

\subsection{Evaluation}

For the evaluation process endocardial and epicardial contours of the datasets $D_{1,2,3,4}$ in end-diastolic and end-systolic phase in all shortaxis MR images are

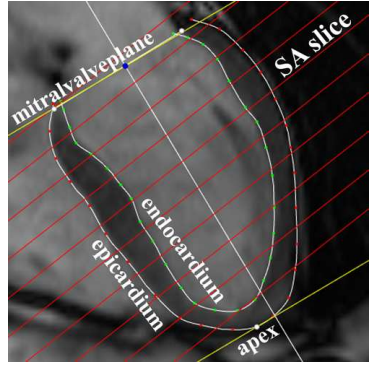

(a)

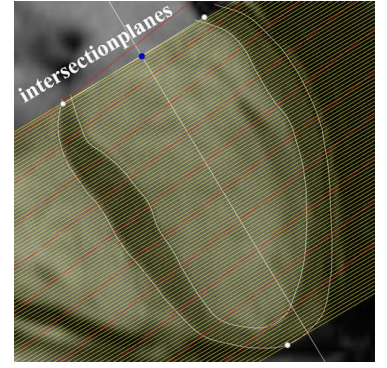

(b)

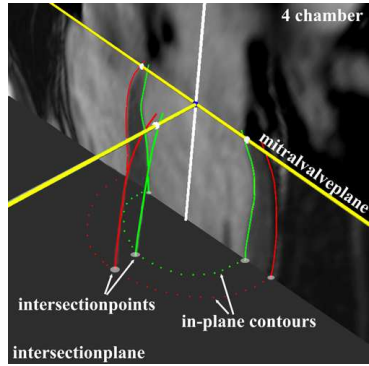

(c)

Fig. 2. Position of the mitral valve plane and SA images (a) and the intersection planes covering the whole LV (b). Intersection points and in-plane contour generation (c) 
outlined by two medical experts using a contour drawing tool [1]. Each observer traced the contours of each dataset two times with the manual segmentation. Therfore approx. 500 contours have to be defined. Based on these contours five global parameters (e.g. end-diastolic Volume (EDV), LV-ejection fraction (LVEF)) were extracted for each dataset using Simpson rule. Then the presented segmentation method is used to compute the same global parameters including LA information. For each dataset only 4 LA contours need to be defined. If needed the shape of the generated model is manually adjusted. To evaluate intraand interobserver difference the mean and standard deviation of the differences of the extracted parameters were compared.

At last the variance of the extracted parameters for MR datasets of the same subject but with different slice thickness $(s=6,8,10,12 \mathrm{~mm})$ are evaluated (Fig. $3(\mathrm{a}))$. Here the parameters for each observer are compared separately to avoid interobserver variability.

\section{Results}

Four 4D Cine MR image sequences were obtained at the Department of Diagnostic and Interventional Radiology from one subject in different positions using a Siemens 1.5T MR scanner. Longaxis and shortaxis images were aquired by using electrocardiographically triggered breath-hold imaging techniques according to the American Heart Assiciation (AHA) scientific statement. For each slice 20 phases provided the complete coverage of the cardiac cycle resulting in a total of 380 cardiac images per sequence. The intra- and interobserver difference comparison between global LV function for manually and semi-automatically segmented images are shown in Tab. 1. The mean difference and standard deviation of all parameters is decreased. In case of intraobserver comparison e.g. the mean EDV difference is reduced from $13.7 \%$ to $2.3 \%$. Concerning slice thickness the mean difference of $\operatorname{EDV}(14.1 \%$ to $5.8 \%)$ and $\operatorname{ESV}(18.8 \%$ to $4.9 \%)$ are decreased but SV $(10.7 \%$ to $20.7 \%)$ and LVEF (4.3\% to $11.6 \%)$ are increased.

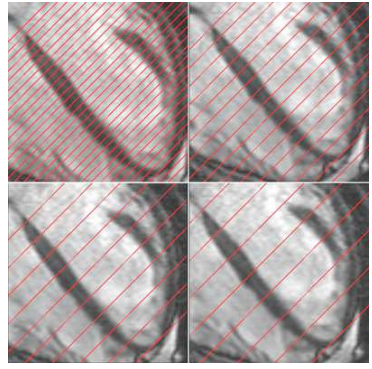

(a)

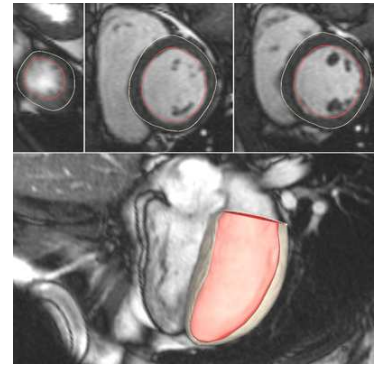

(b)

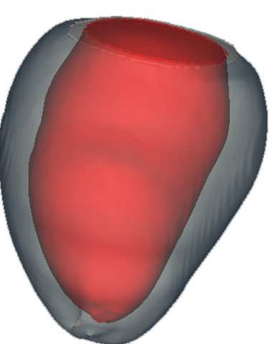

(c)

Fig. 3. LA view of dataset $D_{2}, D_{2}^{1}, D_{3}$ and $D_{4}$. Position of the SA images with different slicethickness $s$ are overlayed (a) and 2D/3D segmentation results (b-c) 
Table 1. Inter- and intraobserver difference comparison between global LV function for manually and semi-automatically segmented images. (EDV: end-diastolic volume; ESV: end-systolic volume; SV: stroke volume; LVEF: left ventricular ejection fraction; Mass: mass of the myocardium)

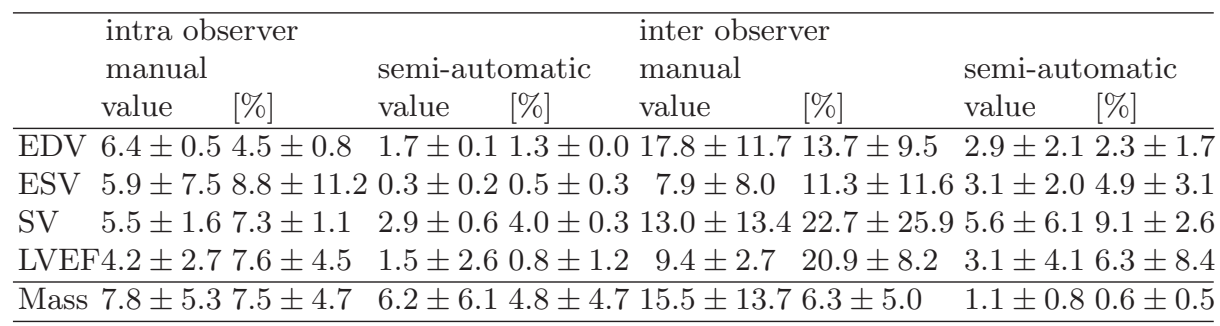

\section{Discussion}

A semi-automatic LV segmentation method has been developed that combines LA information and intersection planes as well as SA images.

In the presented work global parameters including position and orientation of the mitral valve and the apex are extracted. The generation of intersection planes and in-plane contours covering the whole left ventricle enable the construction of a surface model (Fig. $3(\mathrm{~b}+\mathrm{c})$ ). The intra- and interobserver difference can be decreased for all extracted parameters. Using the presented approach the time for the segmentation process is decreased from 60 minutes to 10 minutes including manual adjustment. So the semi-automatic segmentation using LA information proved to be fast and robust for the quantification of LV mass and volume properties.

In future the variability concerning slice thickness has to be evaluated. Also new MR protocols enable the acquisition of a third LA image called 3 chamber view. This image can be used to increase the number of in-plane seedpoints which can improve the quality of the initial surface model.

\section{References}

1. Säring D, et al. Computer-assisted analysis of $4 \mathrm{D}$ cardiac MR image sequences after myocardial infarction. Methods Inf Med. 2006;45(4):377-83.

2. Rueckert D, et al. Shape-based segmentation and tracking in $4 \mathrm{D}$ cardiac MR images. In: CVRMed; 1997. p. 43-52.

3. Spreeuwers LJ, et al. Myocardial boundary extraction using coupled active contours. In: Computers in Cardiology, 2003; 2003. p. 745-8.

4. Goshtasby AA, et al. Fusion of short-axis and long-axis cardiac MR images. Comput Med Imaging Graph. 1996;20(2):77-87.

5. van Geuns RJM, et al. Automatic quantitative left ventricular analysis of cine MR images by using three-dimensional information for contour detection. Radiology. 2006;240(1):215-21.

6. Segars WP, et al. A realistic spline-based dynamic heart phantom. IEEE Trans Nuc Sci. 1999;46(3):503-6. 